\title{
Myoferlin Is a Key Regulator of EGFR Activity in Breast Cancer
}

Andrei Turtoi $^{1,2}$, Arnaud Blomme $^{1}$, Akeila Bellahcène ${ }^{1}$, Christine Gilles ${ }^{3}$, Vincent Hennequière ${ }^{1}$, Paul Peixoto ${ }^{1}$, Elettra Bianchi ${ }^{5}$, Agnès $\mathrm{Noel}^{3}$, Edwin De Pauw ${ }^{2}$, Eric Lifrange ${ }^{4}$, Philippe Delvenne ${ }^{5}$, and Vincent Castronovo ${ }^{1}$

\section{Abstract}

Myoferlin is a member of the ferlin family of proteins that participate in plasma membrane fusion, repair, and endocytosis. While some reports have implicated myoferlin in cancer, the extent of its expression in and contributions to cancer are not well established. In this study, we show that myoferlin is overexpressed in human breast cancers and that it has a critical role in controlling degradation of the epidermal growth factor (EGF) receptor (EGFR) after its activation and internalization in breast cancer cells. Myoferlin depletion blocked EGF-induced cell migration and epithelial-to-mesenchymal transition. Both effects were induced as a result of impaired degradation of phosphorylated EGFR via dysfunctional plasma membrane caveolae and alteration of caveolin homo-oligomerization. In parallel, myoferlin depletion reduced tumor development in a chicken chorioallantoic membrane xenograft model of human breast cancer. Considering the therapeutic significance of EGFR targeting, our findings identify myoferlin as a novel candidate function to target for future drug development. Cancer Res; 73(17); 5438-48. (C2013 AACR.

\section{Introduction}

Epidermal growth factor receptor (EGFR) is a receptor tyrosine kinase (RTK) whose activation and signaling contributes to major cancer key hallmarks including proliferation, invasion, and metastasis (1). As such, EGFR and its downstream signaling is currently one of the most explored pathways in terms of targeting and anticancer treatments. Unfortunately, current therapies aiming to interfere with EGFR signaling components are rather disappointing in clinics, reflecting our limited mechanistic understanding. Today, a wealth of evidence suggests that an essential aspect of EGFR activity regulation consists of receptor internalization (endocytosis) and intracellular targeting (2). This process goes beyond simple recycling of the EGFR and attenuation of the signaling. In fact, it provides an important spatial and temporal component to the activity of the receptor. Endosomes containing EGFR (or other

Authors' Affiliations: ${ }^{1}$ Metastasis Research Laboratory, GIGA Cancer; ${ }^{2}$ Laboratory of Mass Spectrometry, GIGA Systems Biology and Chemical Biology, Department of Chemistry; ${ }^{3}$ Laboratory of Tumor and Development Biology, GIGA Cancer; ${ }^{4}$ Department of Senology, University Hospital (CHU); and ${ }^{5}$ Faculty of Medicine, Department of Anatomy and Pathology, University of Liège, Liège, Belgium

Note: Supplementary data for this article are available at Cancer Research Online (http://cancerres.aacrjournals.org/)

A. Turtoi and A. Blomme have contributed equally to this work.

Corresponding Authors: Vincent Castronovo, Metastasis Research Laboratory, GIGA Cancer, University of Liège, Bat. B23, Liège, 4000 Liège, Belgium. Phone: 32-4366-2479; Fax: 32-4366-2975; E-mail: vcastronovo@ulg.ac.be; and Andrei Turtoi, E-mail: a.turtoi@ulg.ac.be

doi: 10.1158/0008-5472.CAN-13-1142

(C)2013 American Association for Cancer Research. activated RTK), depending of their composition, can either continue to signal in a specific cell compartment or be rapidly degraded in other. At least 2 mechanisms of RTK internalization, respectively, identified as clathrin (CME) or nonclathrin (NCE)-mediated endocytosis, have been described to play essential roles in defining EGFR fate upon ligand-based activation $(2,3)$. The activation of one particular endocytic pathway depends at least in part on EGF concentration (3), and each of the two leads to a different outcome for the receptor (e.g., degradation or recycling). The NCE process is particularly blurry with yet many unknown players that participate/regulate it. Present literature frequently points at caveolin as a protein that is involved in the NCE; however, the presence of this protein is not always a compulsory factor $(2,3)$. This leaves an important question open concerning the identity of proteins participating in NCE. Along these lines, another protein, myoferlin, has been found to colocalize with caveolin and dynamin2 , together being involved in the process of receptor-dependent endocytosis in endothelial cells (4). Although, in normal cells, myoferlin has been described important for cell membrane fusion, repair, and recycling (5-9), this protein has received no attention in the context of RTK internalization in cancer.

Myoferlin is a member of the ferlin family of proteins (including dysferlin and otoferlin as well as 3 additional yet not characterized members FER1L4, FER1L5, and FER1L6) implicated in muscular development (5). Biochemically, myoferlin consists of multiple $\mathrm{C} 2$ domains, which are known to have specialized segments interacting with phospholipids and proteins. In addition, myoferlin has a transmembrane (single pass) and short extracellular domain. In endothelial cells, myoferlin was found to regulate vascular endothelial growth factor receptor 2 (VEGFR2) biologic activity through preventing 
its polyubiquitination and proteasomal degradation (10). In the same cells, myoferlin silencing decreased the expression of angiopoietin-1 receptor (TIE2), another RTK expressed mainly in the vascular endothelium (11). In normal myoblasts, Demonbreun and colleagues showed that myoferlin deficiency leads to a defect in insulin growth factor-like receptor (IGFR1) trafficking as well as to decreased IGFR1 signaling (12). Although increasing number of studies continue to further our understanding of myoferlin function in normal cells, to date, only little (13-15) is known concerning its role in tumor cells. Three previous studies have reported the overexpression of myoferlin at the mRNA level in lung (16) and breast cancer (17) as well as at the protein level in pancreas cancer (18). Our attention has been drawn to this protein owing to the present proteomic study of accessible extracellular and cell membrane proteins in breast cancer tissues, where myoferlin was found as overexpressed in breast cancer cells and absent in normal epithelial cells. This original observation has encouraged us to investigate the possibility that myoferlin overexpression in breast cancer modulates the activity of EGFR. Indeed, we found that myoferlin colocalized with caveolin to control the cellular destiny of activated EGFR. In absence of myoferlin, activated EGFR cannot be degraded in breast cancer cells and hence continues to activate the downstream components of the EGFR pathway, leading to prolonged/aberrant signaling, notably resulting primarily in the inhibition of epithelial-to-mesenchymal transition (EMT) and migration in breast cancer cells.

\section{Materials and Methods}

\section{Patients}

All experiments undertaken with patient material complied with the regulations and ethical guidelines of the University of Liège (Liège, Belgium). All tissue samples were obtained from the Pathology Department of the University Hospital of Liege. The proteomics analysis was conducted using 3 tumoral (ductal adenocarcinoma) and adjacent normal tissue samples, collected from 3 patients. The patients were female, 61 to 78 years of age, and with tumor grade 2 to 3 (Bloome grading). The immunohistochemical (IHC) validation was conducted on a collection of breast cancer tissues comprising 90 tumoral (patients with breast ductal adenocarcinoma, grade 2 and 3 with no known metastases at the time point of surgery) and 10 normal adjacent tissues. The Western blot-based validation was conducted on samples originating from 4 patients with breast ductal and 4 with lobular carcinoma (not included in the mass spectrometry or IHC analysis) as well as their matched normal tissues.

\section{Proteomic analysis}

The analytic approach has been developed and described previously (19). Briefly, fresh human breast cancer biopsies were immediately sliced and soaked in freshly prepared EZ-link Sulfo NHS-SS biotin ( $1 \mathrm{mg} / \mathrm{mL}$, Pierce) solution. Following 20minute incubation at $37^{\circ} \mathrm{C}$, the samples were snap-frozen in liquid nitrogen and converted to powder. Protein extraction was conducted as previously described (19). The protein extracts were mixed with $100 \mu \mathrm{L} / \mathrm{mg}$ streptavidin resin (Pierce) and incubated for 2 hours under rotational conditions at room temperature. The supernatant was retained for the subsequent glycoproteomic analysis (fraction 1) and the streptavidin beads were washed thoroughly. The biotinylated proteins were eluted (fraction 2) using $100 \mathrm{mmol} / \mathrm{L}$ dithiothreitol (DTT; 30 minutes at $60^{\circ} \mathrm{C}$ ). Fraction 1 was also reduced in $100 \mathrm{mmol} / \mathrm{L}$ DTT. Following this, both fractions were alkylated with $150 \mathrm{mmol} / \mathrm{L}$ iodoacetamide (30 minutes at room temperature) and the proteins were then precipitated with $20 \%$ trichloroacetic acid at $4^{\circ} \mathrm{C}$ overnight. Subsequently, the proteins were dissolved (as complete as possible) in $50 \mathrm{mmol} / \mathrm{L} \mathrm{NH}_{4} \mathrm{HCO}_{3}$ and digested using trypsin (Promega; 1:50 protease/protein ratio at $37^{\circ} \mathrm{C}$ ) overnight. The biotinylated peptides (fraction 2) were further processed using mass spectrometry. Fraction 1 was used for the isolation of glycopeptides. For mass spectrometry and data processing analysis, refer to Supplementary Section.

\section{MDA-MB231, MDA-MB468 cell culture, and siRNA- mediated knockdown}

The MDA-MB231 (HTB-26) and MDA-MB468 (HTB-132) cells were obtained from American Type Culture Collection. The cells were authenticated through DNA profiling of 8 different and highly polymorphic short-tandem repeat loci (DSMZ, Braunschweig, Germany). Both cell lines were cultured in Dulbecco's Modified Eagle Medium (DMEM; Life Technologies) supplemented with $10 \%$ heat-inactivated fetal calf serum (MP Biomedicals) and L-Glutamine (MP Biomedicals) at $37^{\circ} \mathrm{C}$, $5 \% \mathrm{CO}_{2}$, and $95 \%$ humidity. The cells were used between passages 5 and 20 reaching near-confluence and were harvested with trypsin. The cells were transfected using Lipofectamine (Lipofectamine 2000 reagent, catalog no. 11668-019, Life Technologies) with small-interfering RNA (Thermo Scientific) directed against myoferlin (siRNA\#1: CCCUGUCUGGAAUGAGA and siRNA\#2: CGGCGGAUGCUGUCAAAUA) or caveolin (CGAGAAGCAAGUGUACGAC) at a concentration of $10 \mathrm{nmol} /$ L/siRNA. ON-TARGETplus Non-Targeting Pool (Dharmacon) was used as a negative control [further referred to as Irrelevant (Irr.) siRNA]. The principal effects (migration, invasion, and EGF stimulation experiments) were evaluated using both myoferlin siRNA oligomers. As both displayed similar levels of silencing, the remaining experiments were conducted using siRNA \# 1 . Sixteen hours after transfection, culture medium was changed and 48 hours later, the cells were lysed and used for all Western blot analyses. For fluorescence-activated cell sorting (FACS), cells were detached with $5 \mathrm{mmol} / \mathrm{L}$ EDTA.

\section{EGF stimulation}

Cells, 32 hours -after transfection, were starved overnight (16 hours) in serum-free DMEM and stimulated with a pulse of $10 \mathrm{ng} / \mathrm{mL}$ of EGF (PeProTech) for different periods of time (up to 120 minutes). Cells were then washed once with PBS and subsequently subjected to protein extraction as described in Supplementary Materials and Methods (under the section Western blot).

\section{Immunofluorescence}

After 16 hours of siRNA-mediated silencing, $5.0 \times 10^{4}$ cells were plated in on a $12 \mathrm{~mm}$ coverslip (Menzer Glaser; 
CB00120RA1) and allowed to grow for additional 36 hours. The cells were then starved overnight in medium without serum and then treated with $10 \mathrm{ng} / \mathrm{mL}$ EGF and allowed to incubate for a maximum of 120 minutes. Treated cells were fixed for 10 minutes at $-20^{\circ} \mathrm{C}$ in a methanol/acetone solution $(80 / 20)$, washed twice with PBS, and blocked in $2 \%$ bovine serum albumin (BSA; Sigma-Aldrich, A3059) for 30 minutes. Coverslips were incubated for 2 hours at room temperature with primary antibodies diluted in $2 \%$ BSA: anti-caveolin (same as for Western blot, see Supplemental Supplementary Data), anti-myoferlin (Santa Cruz Biotechnology; sc-51367), anti-EGFR (Santa Cruz Biotechnology; sc-03), and anti-pEGFR (Millipore; 05-1004). Subsequently, the slides were washed in 3 PBS-BSA washes and incubated with dye-conjugated secondary antibodies (Life Technologies; A-11056, A-11029, and A-21070) for 45 minutes at room temperature. Following 3 additional washes, nuclei were labeled with $2 \mathrm{ng} / \mathrm{mL}$ Hoechst (Merck) for 5 minutes and the coverslips were mounted with Mowiol (Sigma) on glass slides. Images were accrued with laser scanning confocal microscope (AlR, Nikon Instruments).

\section{EGF-mediated EMT assay}

MDA-MB468 cells served as an EGF-inducible EMT model and were described in detail elsewhere (20). Briefly, $48 \mathrm{~h}$ posttransfection, the cells were trypsinized and plated at a confluence of 200,000/well in a 6-well plate. The culture medium was supplemented with EGF for a final concentration of $20 \mathrm{ng} /$ $\mathrm{mL}$. Cells were allowed to grow for further 24 to 48 hours following protein and RNA extraction.

\section{Chicken chorioallantoic membrane (in vivo) tumor assay}

On embryonic day $11,100 \mu \mathrm{L}$ of a suspension of $2 \times 10^{6}$ of MDA-MB231 cells in culture medium mixed (1:1) with Matrigel (BD Biosciences) were deposited in the center of a plastic ring on the chorioallantoic membrane (CAM). Tumors were harvested on embryonic day 18 and were either fixed in $4 \%$ paraformaldehyde solution (30 minutes) for histology analysis or snap frozen in liquid nitrogen for Western blot analysis. Tumor volume was assessed using the formula $\mathrm{V}=\frac{4}{3} \cdot \pi \cdot(H \times L \times W)^{3}$ where $H, L$, and $W$ denote height, length, and width of the tumor.

\section{Statistical analysis}

All experiments were carried out in biologic triplicates. The CAM model was conducted in 3 biologic and 5 technical replicates, where appropriate statistical analysis was conducted using 2-sided Student $t$ test and assuming equal variances. The calculations were conducted with Excel software (Microsoft).

\section{Results}

Proteomic investigation identifies myoferlin as a membrane protein in human breast adenocarcinoma

The proteomic approach used here on 3 human breast cancer samples enriches for extracellular and membrane- bound proteins $(19,21)$. These proteins, which are considered as potentially accessible, are particularly relevant for therapeutic targeting and imaging applications (22). Comparative mass spectrometry analysis (cancer vs. normal adjacent tissue) identified more than 1,400 proteins, of which approximately 800 were already known to be cell membrane-bound or extracellular. The semiquantitative evaluation identified a number of modulated proteins outlined in Supplementary Table S1. The number of identified peptides, Mascot score, and sequence coverage for each of the modulated proteins is outlined in Supplementary Table S2. A number of proteins were identified owing to the glycopeptide enrichment step of the proteomic method used in this study. These are listed in the Supplementary Table S3 along with the glycosylated peptides and the sites of glycosylation that led to their identification.

Myoferlin was identified as a novel breast cancer-related protein in all 3 patient samples used for the proteomic analysis. Next, we sought to confirm myoferlin expression in a larger breast cancer patients' series. We conducted IHC analysis of myoferlin expression in 90 breast adenocarcinoma samples and in 10 adjacent normal breast tissues. The IHC analysis (Fig. 1A) fully confirmed the mass spectrometry data and showed that myoferlin overexpression is a consistent feature of breast cancer. The staining was specifically detected in tumor cells, mostly conferred not only to the plasma membrane but also the cytoplasm. Normal adjacent breast ducts were predominantly negative, with low/moderate positivity detectable mainly in endothelial cells (Fig. 1A). Further Western blot analysis (Fig. 1B) on paired samples (tumor and adjacent normal tissue from same individual) of breast ductal adenocarcinoma and lobular carcinoma confirmed IHC data. We then conducted a Western blot evaluation of myoferlin expression in a selection of normal human tissues. Interestingly, myoferlin expression was not detectable in all the normal tissues tested (Fig. 1C), except testis and skin.

\section{Myoferlin silencing in breast cancer cells impaired EGF- induced migration and EMT}

EGFR pathway signaling has pronounced cellular consequences in cancer leading to differentiation and alterations of associated processes such as migration, invasion, and EMT (23-25). Previous studies have shown that MDA-MB231 cells are responsive to EGF stimulus, characterized by enhanced migration (25). We have examined whether MDA-MB231 cells lacking myoferlin would still show responsiveness toward EGF (Fig. 2A), and found that myoferlindepleted cells are unable to migrate (Boyden chamber assay) when pulsed with EGF. This impaired migration toward EGF was also observable in myoferlin-depleted MDA-MB468 breast cancer cells (Supplementary Data and Supplementary Fig. S2).

To further examine the consequence of myoferlin depletion on EGFR signaling and related biologic processes, we used an EGF-inducible EMT breast cancer cell model. In our recent work (20), we have shown that MDA-MB468 cells are able to undergo EMT in vitro following EGF stimulation. Unlike MDA-MB231 cells, which in basal conditions are displaying a prominent mesenchymal appearance, the 


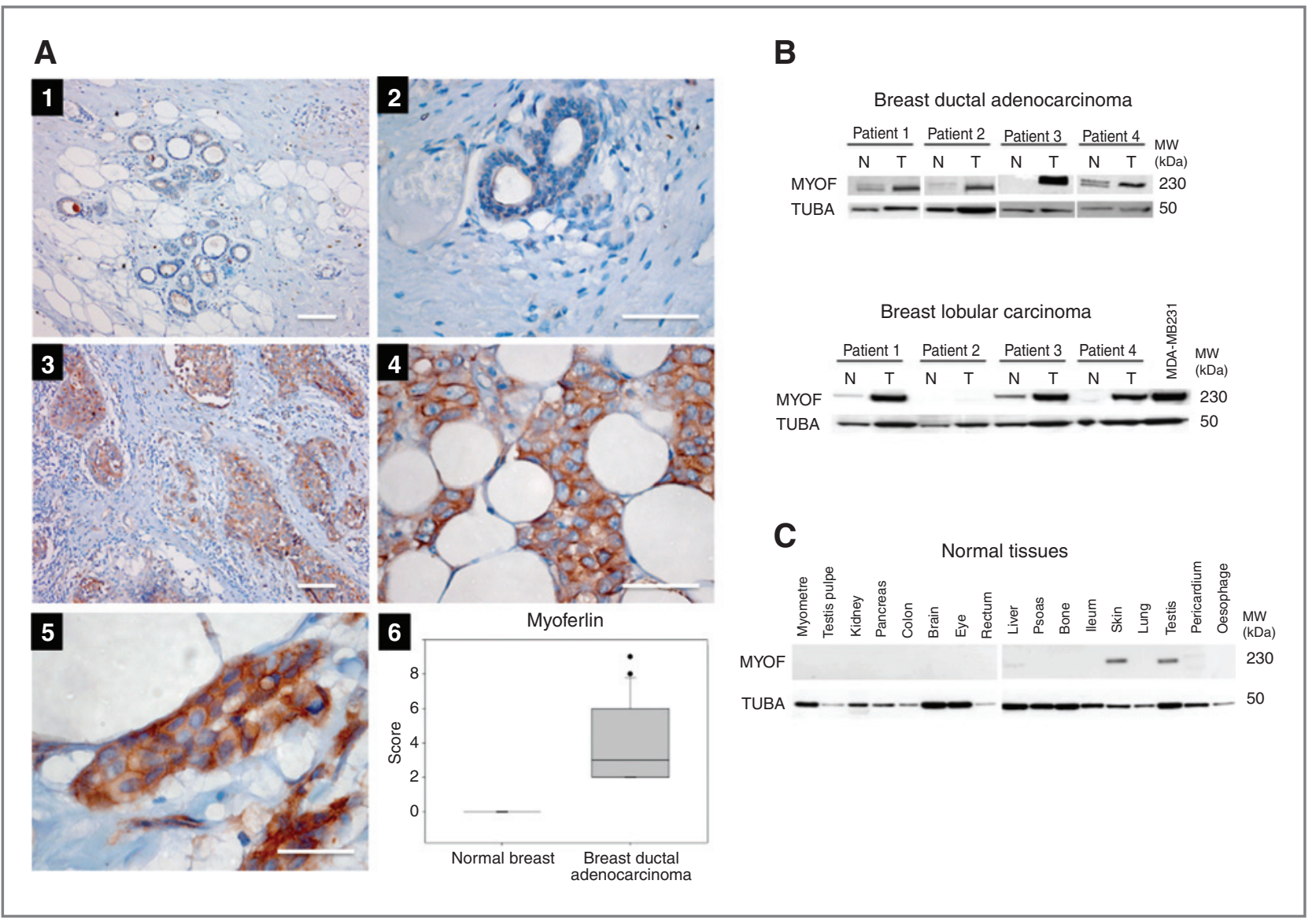

Figure 1. Myoferlin is overexpressed in human breast cancer tissue. A, representative immunohistochemical staining of myoferlin (MYOF) expression in human breast malignant tumors; normal breast ducts did not express/had low levels of myoferlin (1-2); breast cancer cells (in situ; 3) as well as cancer cells invading breast fat tissue (4) are both expressing myoferlin; the expression pattern corresponded to a cytoplasm/membrane positivity (5); semiquantitative evaluation (6), as described in Material and Methods, of myoferlin expression in 90 patients with breast ductal adenocarcinoma showed positivity in all examined cases. Magnification used, $\times 100$ ( 1 and 3 ); $\times 400$ ( 2 and 4); and $\times 600$ (5); scale bar, $50 \mu \mathrm{m}$. B, Western blot analysis of myoferlin expression in 4 matched (tumor/normal) ductal and 4 lobular breast carcinoma patients. C, Western blot evaluation of myoferlin expression in a selection of normal human tissues.

MDA-MB468 cells are epithelial-like. In presence of the EGF stimulus, MDA-MB468 cells undergo EMT, adopting a mesenchymal phenotype that is primarily characterized by a strong induction of vimentin (VIM) expression coupled to the downregulation of E-cadherin (CDH1) as well as by modulation of other more specific EMT markers (further detailed in ref. 20). In the present work, we used VIM and CDH1 as surrogate markers of EGF-induced EMT in the MDA-MB468 cells. Myoferlin depletion in MDA-MB468 cells significantly impaired their capacity to undergo EGFinduced EMT with both reduced VIM induction and downregulation of $\mathrm{CDH1}$ expression (Fig. 2B and C). These effects were already noticeable 24 hours post-EGF treatment (VIM) and became more pronounced at 48 hours (VIM and $C D H 1$ ).

\section{Myoferlin regulates EGFR fate upon EGF-mediated receptor activation}

Following ligand binding, EGFR undergoes phosphorylation, internalization, and intracellular targeting to modulate downstream signaling. We have investigated whether myoferlin is involved in the EGFR signaling machinery. As shown in Fig. 3A, upon EGF stimulation, depletion of myoferlin in MDA-MB231 cells led to sustained pEGFR activation, evidenced through prominent phosphorylation of Y1173 residue (the same effect was observed in MDA-MB468, Supplementary Fig. S2). Following this, we examined whether the downstream targets of the EGFR signaling were also activated. One major key component of the pathway is AKT (RAC-alpha serine/threonine-protein kinase), which is readily phosphorylated upon EGF stimulation. The results in Fig. 3B show that EGF stimulation of MDAMB231 cells induced the phosphorylation of AKT on S473 and that this signaling pathway is enhanced and prolonged in myoferlin-depleted cells.

As shown in the Fig. 3A, total EGFR expression levels were increased in comparison with the mock-transfected cells. Further gene expression analysis showed moderately elevated levels of EGFR mRNA (1.5-fold in comparison with the siRNA Irr. at 48 hours) evident in basal condition and further observed during EGF stimulation (data not shown). Although the gene expression and protein synthesis levels are not directly 
A EGF Induced migration - MDA-MB231

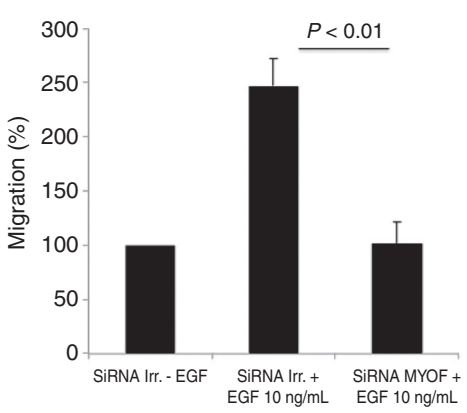

C

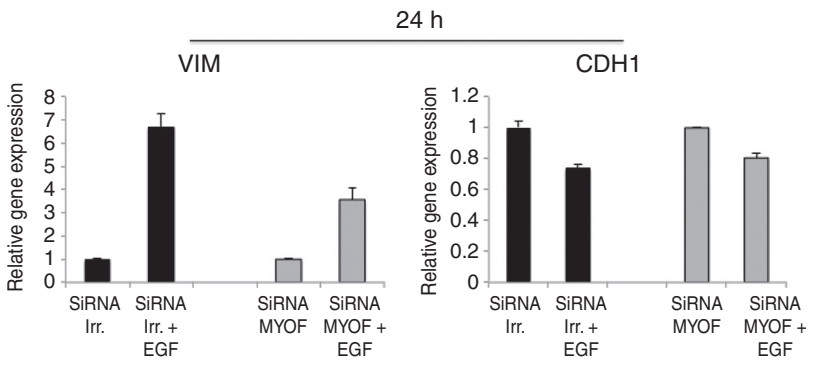

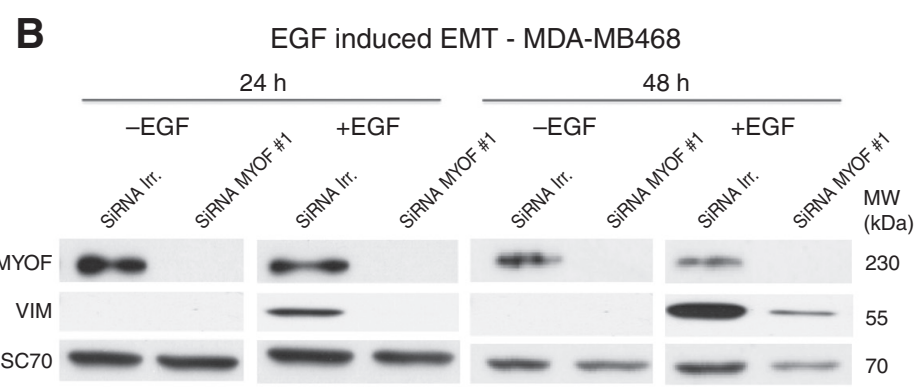

EGF induced EMT - MDA-MB468

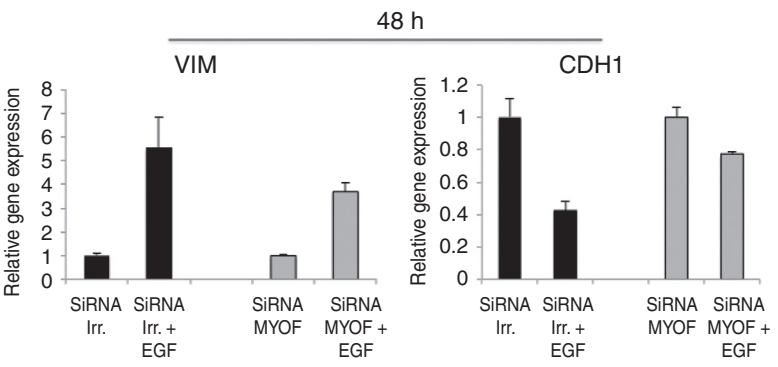

Figure 2. Myoferlin depletion inhibits EGF-induced migration and EMT in breast cancer cells. A, EGF-induced MDA-MB231 cell migration is stalled in the absence of MYOF (similar data are shown with MDA-MB468; Supplementary Fig. S2). Error bars indicate SD of means from 3 independent biologic replicates. Statistical significance $(P)$ was evaluated using an unpaired Student $t$ test (for details see Materials and Methods). B, myoferlin is essential for EGF-induced EMT in MDA-MB468 breast cancer cells. EGF-mediated vimentin induction is impeded in MYOF-depleted MDA-MB468 cells (Western blot analysis). Displayed is one representative experiment of 3 biologic replicates. C, qRT-PCR analysis of CDH1 and VIM gene expression in EGF-treated MDA-MB468 cells at 24 and 48 hours post-MYOF silencing. Error bars indicate SD of means from 3 independent biologic replicates.

comparable, it is unlikely that this moderate increase in EGFR mRNA can justify the prominent increase of protein levels. To further explore this effect, we next verified whether the internalization process was affected. Quantitative measurement of EGFR levels on the surface of living MDA-MB231 cells during EGF stimulation revealed comparable internalization kinetics between myoferlin-depleted and control conditions (Fig. 3C). Further immunofluorescence-based analysis showed that the EGFR was internalized in myoferlin-depleted cells in the same manner as in the control condition. However, after 60 and 120 minutes, the EGFR foci failed to resolve in the absence of myoferlin (Fig. 3D). These observations suggest that the elevated EGFR levels in myoferlin-depleted cells are probably linked to their inability to properly degrade the receptor and hence switch off the pEGFR signal. To test whether the blocking of the EGFR degradation will show similar effect on pEGFR levels as myoferlin silencing, we inhibited proteolytic activity of the proteasome using MG132 while subjecting the cells to the same EGF stimulation as shown in Fig. 3A. The comparison of the resulting EGFR/pEGFR expression patterns (Supplementary Fig. S3) showed that impairment of proteasome degradation caused a sustained EGFR expression and activation, similar to what is observed when myoferlin is silenced. Finally, we examined whether myoferlin is colocalizing with EGFR in basal conditions and in the presence of EGF (Fig. 3E). The data show that EGFR does colocalize with myoferlin in MDA-MB231 cells, both in basal and EGF conditions. As displayed in the Fig. 3E, myoferlin and EGFR are also detectable and colocalized in the endosomic vesicles, structures typically observed following EGF-induced EGFR endocytosis. Considering the existence of 2 EGFR internalization mechanisms (CME and NCE), we next verified whether clathrin or/and caveolin are affected following myoferlin silencing in MDA-MB231 cells.

\section{Myoferlin does not affect clathrin cellular distribution/ quantity in MDA-MB231 cells}

Immunofluorescence analysis showed that myoferlin colocalizes with clathrin in breast cancer cells both in serum and EGF-rich conditions (Supplementary Fig. S6A). As expected, clathrin showed evidence of colocalization with pEGFR during EGF-stimulation (Supplementary Fig. S6B). However, myoferlin depletion neither induced any marked modification of clathrin distribution in the cell (Supplementary Fig. S6B) nor a change in clathrin total protein levels (Supplementary Fig. S6C).

\section{Myoferlin is critical for proper assembly of caveolin in caveolae}

Previous studies reported the existence of an intimate relationship between caveolin (and particularly the caveolae) and myoferlin in the context of endocytosis (9). In 


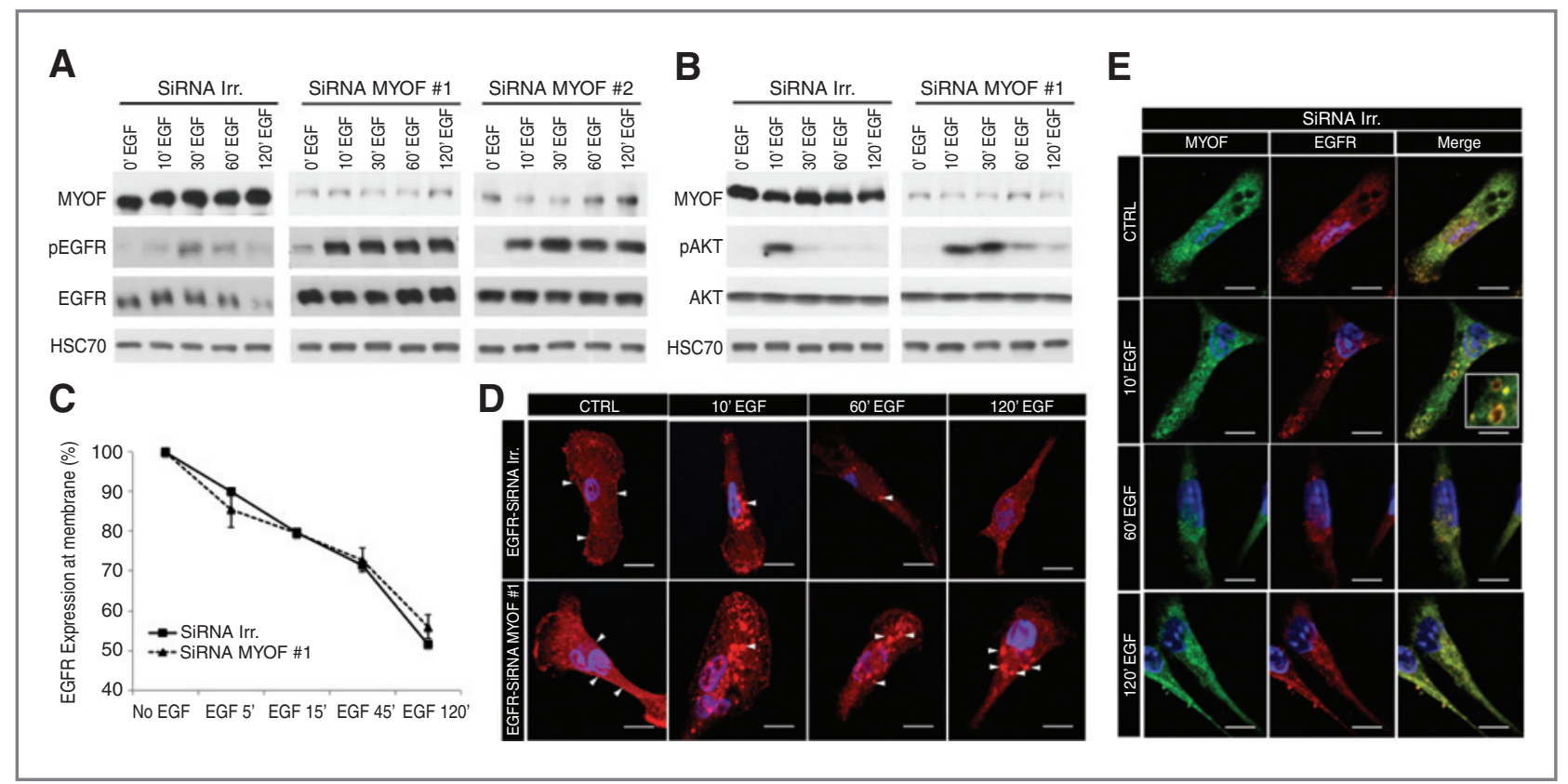

Figure 3. Myoferlin depletion in MDA-MB231 cells results in sustained EGFR phosphorylation upon EGF stimulation. A, Western blot analysis of time-dependent pEGFR and EGFR expression levels following EGF stimulation and myoferlin depletion 48 hours posttransfection. Two different siRNAs directed against myoferlin were used. B, Western Blot analysis of time-dependent AKT/pAKT expression following EGF stimulation and myoferlin silencing (at 48 hours after transfection). C, FACS analysis of EGFR surface levels (intact cells) following time-dependent EGF stimulation of myoferlin-depleted MDA-MB231 cells. Error bars in A and B indicate SD of means from 3 independent biologic experiments. D, EGFR immunofluorescence analysis of MDA-MB231 cells following MYOF depletion and EGF stimulation. White arrows point at EGFR, which at later time-points is internalized and visible as clusters. E, EGFR/MYOF immunofluorescence analysis of MDA-MB231 cells following MYOF depletion and EGF stimulation. $D$ and E, the control condition (CTRL) was not treated with EGF. All images were taken at $\times 400$ magnification, identical setting of photomultipliers, and are representative of at least 3 biologic replicates; scale bars, $5 \mu \mathrm{m}$.

accordance with literature, we found that myoferlin was colocalized with caveolin both in control (basal condition) as well as in EGF-treated cells along the treatment time course (Fig. 4A). Further immunofluorescence analysis of pEGFR and caveolin in EGF-stimulated MDA-MB231 cells (Fig. 4B) showed evidence of colocalization of both proteins in mock-transfected cells, both in basal and EGF-stimulated conditions. In myoferlin-depleted cells, the colocalization was weaker and at latter time points (60 and 120 minutes) pEGFR and caveolin did not colocalize. We also observed that caveolin foci in myoferlin-depleted cells were larger and more variable in size, which is particularly visible at later time points following EGF stimulation (Fig. 5A). Moreover, myoferlin silencing in nonstimulated MDA-MB231 cells resulted in a higher concentration of caveolin homo-oligomers (Fig. 5B, left), the presence of major aberrant molecular weight forms (in comparison with the control condition), and an enhanced enrichment of caveolin in lipid-raft microdomains (Fig. 5B, right). The latter pool of caveolin was not found in the same fractions where myoferlin was present. The appearance of caveolin homo-oligomers with aberrant molecular weight was particularly emphasized after EGF stimulation in myoferlin-depleted cells (Fig. 5C). The present data suggest that the interaction between myoferlin and caveolin is essential for proper organization of caveole and endocytosis of pEGFR. This is further supported by the data shown in Fig. 5D, where siRNA-mediated depletion of caveo- lin in MDA-MB231 cells, similar to myoferlin silencing, induced an accumulation of pEGFR. In contrast, caveolin depletion was unable to inhibit the degradation of the EGFR upon EGF stimulation. Phospho-EGFR levels, although initially elevated, returned to basal levels at later stage.

Myoferlin affects migration, invasion, and survival of tumor cells and is essential for tumor growth in vivo

In vitro studies in basal conditions (medium with serum) show that myoferlin depletion induces reduction in cell migration $(-50 \%)$ and invasion $(-60 \%)$ but does not affect cell proliferation (Supplementary Fig. S1B and S1C). In addition, myoferlin depletion leads to a modest reduction of cell growth in vitro (10\%-20\%; Supplementary Fig. S1D). Keeping in mind that tumors are rich in growth factors (e.g., EGF), we next sought to understand whether EGF stimulation of myoferlindepleted MDA-MB231 cells might further potentiate the cell death. Assaying for apoptosis showed that myoferlin-depleted MDA-MB231 cells show up to a 3-fold increase of cell death following EGF stimulation (Fig. 6B). Starting from the present in vitro data, we next sought to determine whether the loss of myoferlin in breast cancer cells had an impact on tumor growth in vivo. We implanted MDA-MB231 cells onto the chorioallantoic membrane (CAM) of the chicken embryos and evaluated the tumor development when myoferlin was depleted (Fig. 6C). As shown in the Fig. 6A, siRNA-mediated myoferlin depletion is highly effective, lasting for the entire time period of 


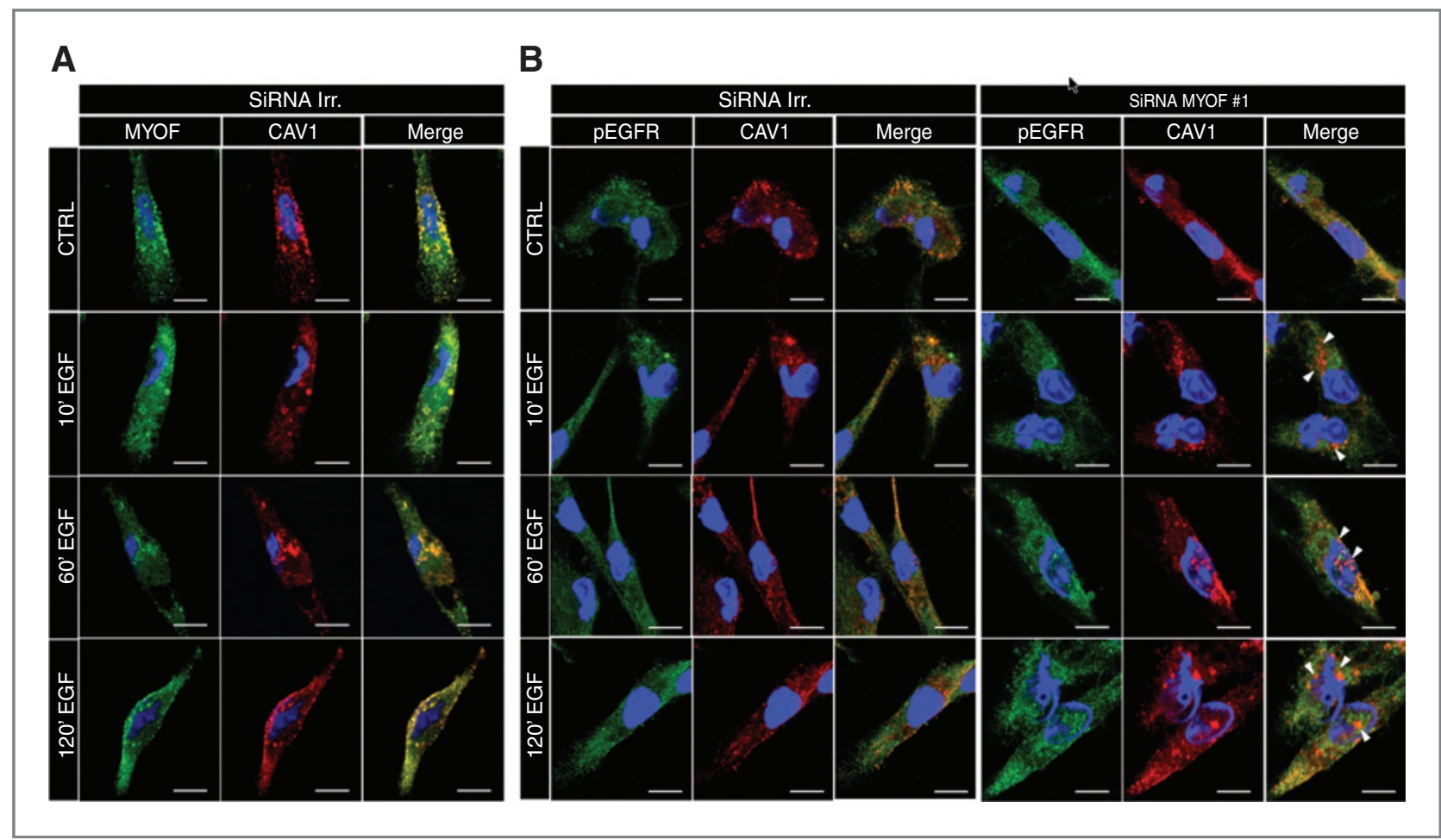

Figure 4. Caveolin colocalizes with myoferlin and pEGFR during EGF-mediated receptor activation. A, immunofluorescence analysis of MYOF and caveolin (CAV1) colocalization in basal conditions (CTRL) and during EGF stimulation. Displayed are mock-transfected cells. B, immunofluorescence analysis of CAV1-pEGFR colocalization during EGF-mediated receptor activation in myoferlin-deficient MDA-MB231 cells 48-hour posttransfection. White arrows indicate examples of several major differences in localization of the 2 proteins. All images were accrued at $\times 400$ magnification, identical setting of photomultipliers, and are representative of at least 3 biologic replicates; scale bars, $5 \mu \mathrm{m}$.

tumor growth in the present model. On day 7 postimplantation, tumor volumes were measured and showed that myoferlin depletion halved the tumor volume (reduction by $40 \%$ ) within this short time period (Fig. 6C). Histologic analysis of myoferlin-depleted tumors showed general decrease of cellularity characterized by loosely associated cells, lacking orientation typically observed in tissue structures (Fig. 6D). IHC staining of EGFR expression in mock-transfected and myoferlin-depleted tumors showed a stronger EGFR immunostaining in myoferlin-depleted tumor cells. The EGFR staining pattern was predominantly homogeneous in mock-transfected tumor cells, whereas myoferlin-depleted cells often displayed higher accumulation of EGFR, similar to the one observed in vitro post-EGF stimulation (Fig. 3D).

\section{Discussion}

EGFR is one of the receptors most commonly associated with human tumors. Ligand binding and dimerization causes autophosphorylation of the intracytoplasmic domains and activation of the intracellular tyrosine kinase. Activated EGFR (pEGFR) stimulates a number of different signal transduction pathways, which play important roles in various cellular processes such as cell proliferation and migration. Our study shows for the first time that myoferlin is overexpressed at the protein level in all human breast adenocarcinomas analyzed and that myoferlin knockdown in breast cancer cells interferes with EGF-induced cell migration and EMT. Moreover, our data designate an unexpected link between myoferlin and EGFR in breast cancer cells, where myoferlin intervenes in the control of EGFR activity and signaling. Consistent with the previous report of Bernatchez and colleagues (9) in endothelial cells, we show that myoferlin colocalizes with caveolin in breast cancer cells, both in basal condition and upon EGF stimulation. As the major component of membrane caveolae, caveolin interacts with a variety of partners such as integrins and RTK receptors and functions as a regulatory platform for their respective postligand signaling (26). Because of the frequent deletion of caveolin gene in cancers, including breast cancer, caveolin has been considered as a potential tumor suppressor (27). Caveolin scaffolding has been previously shown to induce EGFR sequestration in tumor cells. In particular, Lajoie and colleagues (28) propose that the sensitivity of cancer cells to EGF stimulus can be controlled by 2 membrane domains: on one hand, caveolin that concentrates EGFR and blocks it from signaling and on the other hand, the galectin lattice that holds EGFR at the membrane and favors its mitogenic signaling. Despite the prevailing evidence that caveolin acts as a tumor suppressor, it is noteworthy that its overexpression has been also associated with the metastatic potential of lung and prostate cancer cells (29-31). This study provides an answer to these apparently contradictory findings by pointing for the first time to myoferlin as an important regulator of caveolin scaffold in breast cancer cells. We show that myoferlin suppression results in enhanced clustering of 


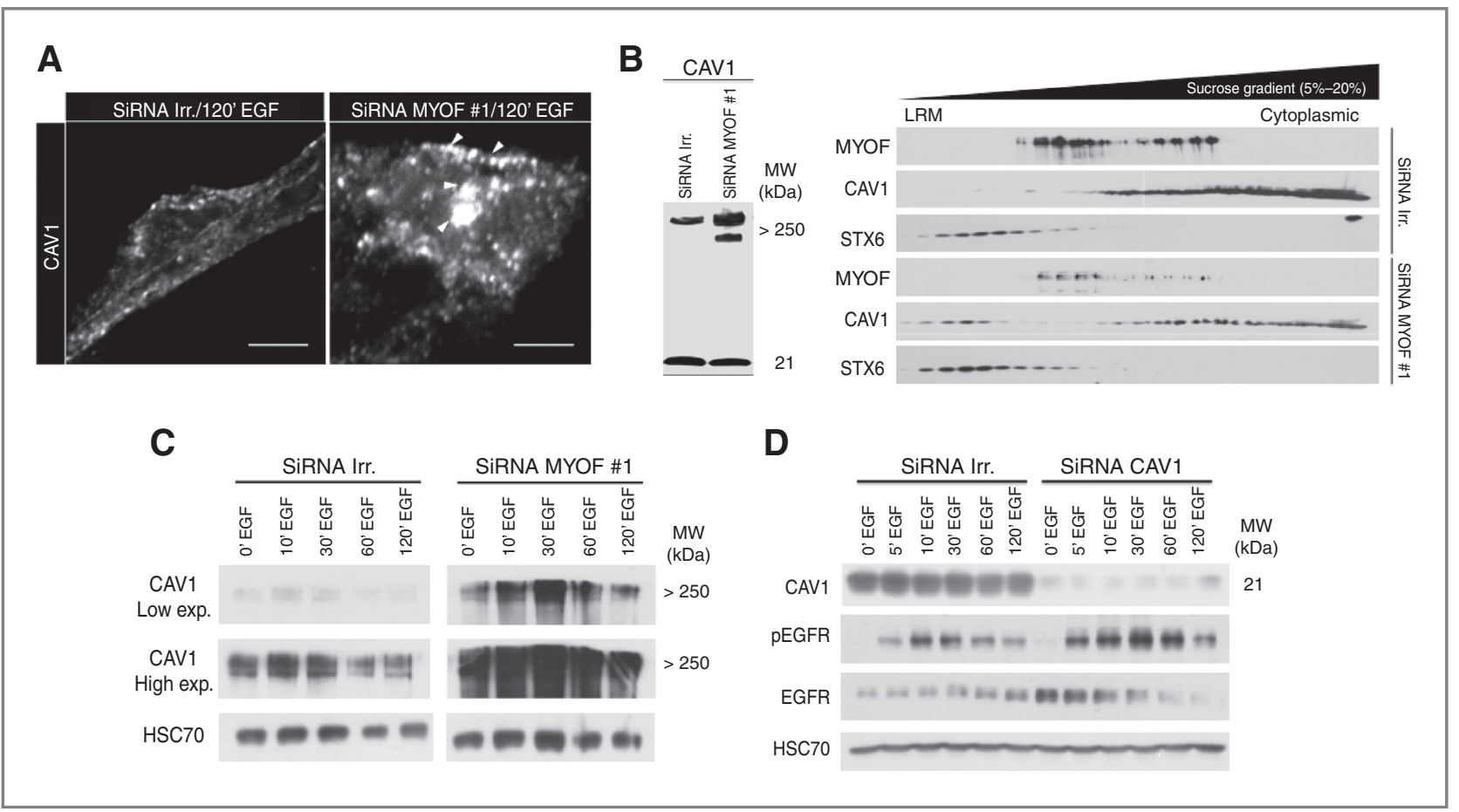

Figure 5. Myoferlin affects the functional assembly of caveolin into caveolae. A, immunofluorescence analysis of CAV1 in myoferlin-deficient MDA-MB231 cells 120-minute post-EGF stimulation. Arrows indicate large CAV1 clusters accumulating in the myoferlin-depleted condition. Magnification, $\times 400$; scale bars, $5 \mu \mathrm{m}$. B, left, Western blot analysis of CAV1 in nondenaturing conditions (with or without MYOF depletion) shows high-molecular weight complexes corresponding to CAV1 homomultimers. Right, sucrose-gradient fractionation of CAV1 complexes in MYOF-depleted MDA-MD231 cells; LRM, lipid-raft microdomain fraction. Syntaxin 6 (STX6) is marker of lipid rafts. C, time-kinetics of high-molecular weight (>230 kDa) CAV1 expression (homomultimers) in MDA-MB231 cells following MYOF depletion and EGF stimulation. D, CAV1 depletion in MDA-MB231 cells leads to a transient accumulation of pEGFR upon EGF stimulation 48-hour posttransfection. Displayed are CAV1 monomers at approximately $21 \mathrm{kDa}$. All data are representative experiments from 3 biologic replicates (A-D).

caveolin oligomers in lipid-raft microdomains as well as the emergence of smaller oligomers of aberrant size. Our data notably show that myoferlin (but not caveolin) depletion is sufficient to block EGFR degradation and to stop the attenuation of EGFR phosphorylation upon EGF stimulation. We found that in breast cancer cells myoferlin depletion does not affect EGFR endocytosis rate, suggesting that this protein is dispensable at this early step. As the internalization proceeds, the cargo (in this case EGFR) needs to be targeted in the cell for activating downstream effectors and subsequently to be neutralized via proteasomal and lysosomal degradation (2). Previous studies suggest that proteasomal degradation precedes and is necessary for the lysosomal targeting of the receptor (32-34). A recent multiparametric image analysis study reveals a predominant role of caveolin in endosomal progression rather than internalization of EGFR (35). Altogether, these observations suggest that myoferlin depletion favors caveolin oligomers scaffold formation, which sequesters EGFR and interferes with the normal ligand-induced degradation of EGFR. We note that this mechanistic scenario applies to EGFR in breast cancer cells and that in other cell types myoferlin may differently regulate the fate of other RTKs, as previously shown with VEGFR2 in endothelial cells (10).

Our results indicate that permanent phosphorylation of EGFR in cells lacking myoferlin has detrimental effects on the EGFR-controlled biologic processes. We believe that the length of signaling has an impact on which cellular processes are activated downstream. Same is the case with Raf kinase (36), where high-intensity Raf signal causes cell-cycle arrest; this is in contrast to the normally assumed proproliferation function. Recently, Rush and colleagues (37) have shown that high endosomal accumulation of activated EGFR induces apoptosis in MDA-MB468 cells. The authors have used monensin to block endosomal trafficking of EGFR. As EGFR accumulation and activation pattern shown in the current study largely exceeds the magnitude of EGFR accumulation shown previously (37), it is reasonable to propose that cell death is probably the terminal outcome of myoferlin depletion. The present results confirm this and show a strong induction of apoptosis in myoferlin deficient, EGF-stimulated cells. This could be the key factor for explaining the significant reduction in size of myoferlin-depleted MDAMB231 tumors in vivo. One of the most striking biologic effects observed here was the inability of breast cancer cells to respond to EGF-stimulus to migrate (MDA-MB231 and MDA-MB468) or undergo EMT (MDA-MB468). Li and colleagues (14) reported recently that MDA-MB231 underwent a spontaneous reverse EMT in the absence of myoferlin. However, similar data published by the same authors elsewhere (38) partially dispute their recently published results (e.g., vimentin levels after myoferlin depletion; ref. 14). We were unable to observe similar effects in MDA- 


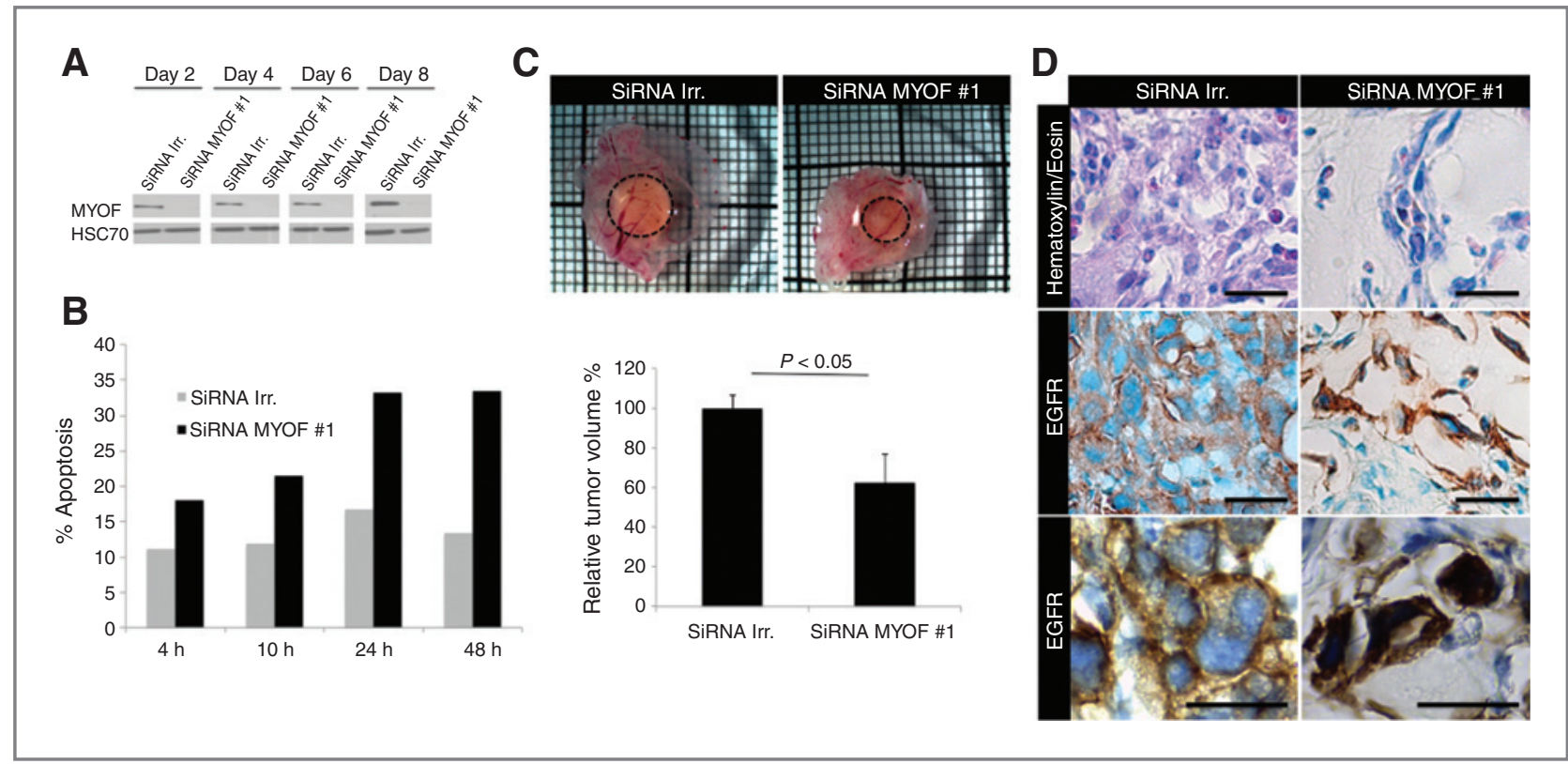

Figure 6. Myoferlin depletion reduces breast cancer tumor volume in vivo. A, Western blot analysis of MYOF expression in MDA-MB231 cells up to 8 days posttransfection (in vitro). B, apoptosis induction (Annexin-V assay) at several time points and following EGF stimulation of MYOF-depleted MDA-MB231 cells (in vitro). C, effect of MYOF silencing in MDA-MB231 CAM tumor model. Tumors collected at day 7 of tumor development were measured in volume (outlined in Materials and Methods; top). Displayed is macroscopic tumor appearance in both conditions. Representative images of 3 biologic and 5 technical replicates. Quantitative evaluation of the tumor volume showed a significant decrease in the MYOF-depleted MDA-MB231 tumors (bottom). Error bars indicate SD of means from 3 independent biologic replicates with 5 tumors per condition. Statistical significance $(P)$ was evaluated using an unpaired Student $t$ test. D, histologic evaluation of paraffin-embedded CAM tumors. Top, hematoxylin and eosin staining of the tumor sections show a decreased cellularity in myoferlin-depleted tumors. Pictures were taken at $\times 400$ magnification. Middle, EGFR expression in tumor cells from control and myoferlindepleted CAM tumors (magnification, $\times 400)$. Bottom, a higher magnification $(\times 900)$ of cells stained for EGFR expression in control and myoferlin-depleted CAM tumors. Data are representative experiments from 3 biologic replicates; scale bars correspond to $20 \mu \mathrm{m}$.

MB231 cells as shown in ref. 14; neither cell attachment nor vimentin levels are affected upon myoferlin silencing (detailed in Supplementary Data, Fig. S5). Interestingly, Li and colleagues (14) and Eisenberg and colleagues (13) both showed that short hairpin RNA (shRNA)-mediated myoferlin depletion impairs MDA-MB231 cells' invasion; however, it has no effect on cell migration or proliferation. Our invasion and proliferation data are in agreement with the literature $(13,14)$. However, opposite to the previous findings $(13,14)$, we show that myoferlin silencing exhibits a strong inhibitory effect on the ability of MDA-MB231 cells to migrate. Although unable at this stage to explain for these discrepancies, we think that experimental differences and adaptation processes when using shRNAs $(13,14)$ in contrast with siRNAs (present study) might play an important role. First, previous studies $(13,14)$ did not use any coating of Transwell inserts when conducting migration experiments; we have used denatured protein matrix coating (gelatin) to provide environment for the cells to attach and subsequently migrate through the insert. Second, adaptation processes may arise with stable transfected cell lines, which in turn may mask the true phenotype $(39,40)$. Concomitantly with our research, Leung and colleagues (15) have reported on myoferlin function in mouse Lewis lung carcinoma cells (mLLC). In accordance with our in vivo data, the authors have showed that myoferlin has a strong impact on tumor growth in vivo. The authors have assessed both cell prolif- eration and apoptosis (in vitro and in vivo) and found that myoferlin silencing impacted mainly proliferation and not apoptosis in mLLC cells. These interesting data in a murine system strengthen the role of myoferlin as an oncogene protein but also indicate that myoferlin may assume similar yet distinct roles in tumors of different types. In conclusion, our data show that myoferlin is overexpressed in a large cohort of human breast cancer and that silencing of myoferlin impedes breast cancer cells to respond to EGF-mediated protumoral stimulus. The latter occurs at least in part owing to the aberrant caveolin oligomerization.

\section{Disclosure of Potential Conflicts of Interest \\ No potential conflicts of interest were disclosed.}

\section{Authors' Contributions}

Conception and design: A. Turtoi, A. Bellahcene, P. Peixoto, V. Castronovo Development of methodology: A. Turtoi, A. Blomme, A. Bellahcene, C. Gilles, P. Peixoto, E. De Pauw

Acquisition of data (provided animals, acquired and managed patients, provided facilities, etc.): A. Turtoi, A. Blomme, C. Gilles, V. Hennequière, P. Peixoto, E. De Pauw, E. Lifrange, P. Delvenne

Analysis and interpretation of data (e.g., statistical analysis, biostatistics, computational analysis): A. Turtoi, A. Blomme, V. Hennequière, P. Peixoto, A. Noel, P. Delvenne

Writing, review, and/or revision of the manuscript: A. Turtoi, A. Blomme, A Bellahcene, C. Gilles, A. Noel, E. De Pauw, E. Lifrange, P. Delvenne, V. Castronovo Administrative, technical, or material support (i.e., reporting or organizing data, constructing databases): $A$. Turtoi, V. Hennequière, $P$. Delvenne Study supervision: A. Turtoi, P. Peixoto, P. Delvenne, V. Castronovo

Evaluated immunohistochemical staining: E. Bianchi 


\section{Acknowledgments}

The authors thank GIGA-Proteomics and GIGA-Imaging Platforms as well as ULG Biobank for experimental support. The authors also thank Evgenia Turtoi and Pascale Heneaux for IHC experiments as well as Dr. Pamela Maris for the BrdU data.

\section{Grant Support}

This work was supported by a grant from the Research Concerted Action (IDEA project) of the University of Liège (ULG), Belgium, from the National Fund

\section{References}

1. Yarden $Y$, Pines G. The ERBB network: at last, cancer therapy meets systems biology. Nat Rev Cancer 2012;12:553-63.

2. Sigismund S, Woelk T, Puri C, Maspero E, Tacchetti C, Transidico P, et al. Clathrin-independent endocytosis of ubiquitinated cargos. Proc Natl Acad Sci U S A 2005;102:2760-5.

3. Sigismund S, Argenzio E, Tosoni D, Cavallaro E, Polo S, Di Fiore PP. Clathrin-mediated internalization is essential for sustained EGFR signaling but dispensable for degradation. Dev Cell 2008;15: 209-19.

4. Davis DB, Delmonte AJ, Ly CT, McNally EM. Myoferlin, a candidate gene and potential modifier of muscular dystrophy. Hum Mol Genet 2000;9:217-26.

5. Doherty KR, Demonbreun AR, Wallace GQ, Cave A, Posey AD, Heretis $\mathrm{K}$, et al. The endocytic recycling protein EHD2 interacts with myoferlin to regulate myoblast fusion. J Biol Chem 2008;283:20252-60.

6. Posey AD Jr, Demonbreun A, McNally EM. Ferlin proteins in myoblast fusion and muscle growth. Curr Top Dev Biol 2011;96:203-30.

7. Martens S, McMahon HT. Mechanisms of membrane fusion: disparate players and common principles. Nat Rev Mol Cell Biol 2008; 9:543-56.

8. McNeil PL, Kirchhausen T. An emergency response team for membrane repair. Nat Rev Mol Cell Biol 2005;6:499-505.

9. Bernatchez PN, Sharma A, Kodaman P, Sessa WC. Myoferlin is critical for endocytosis in endothelial cells. Am J Physiol Cell Physiol 2009;297:C484-92.

10. Bernatchez PN, Acevedo L, Fernandez-Hernando C, Murata T, Chalouni C, Kim J, et al. Myoferlin regulates vascular endothelial growth factor receptor-2 stability and function. J Biol Chem 2007;282: 30745-53.

11. Yu C, Sharma A, Trane A, Utokaparch S, Leung C, Bernatchez P. Myoferlin gene silencing decreases Tie-2 expression in vitro and angiogenesis in vivo. Vascul Pharmacol 2011;55:26-33.

12. Demonbreun AR, Posey AD, Heretis K, Swaggart KA, Earley JU, Pytel $P$, et al. Myoferlin is required for insulin-like growth factor response and muscle growth. FASEB J 2010;24:1284-95.

13. Eisenberg MC, Kim Y, Li R, Ackerman WE, Kniss DA, Friedman A. Mechanistic modeling of the effects of myoferlin on tumor cell invasion. Proc Natl Acad Sci U S A 2011;108:20078-83.

14. Li R, Ackerman WE, Mihai C, Volakis LI, Ghadiali S, Kniss DA. Myoferlin depletion in breast cancer cells promotes mesenchymal to epithelial shape change and stalls invasion. PLoS ONE 2012;7:e39766.

15. Leung C, Yu C, Lin Ml, Tognon C, Bernatchez P. Expression of myoferlin in human and murine carcinoma tumors: role in membrane repair, cell proliferation, and tumorigenesis. Am J Pathol 2013;182: 1900-9.

16. Difilippantonio $S$, Chen $Y$, Pietas $A$, Schlüns $K$, Pacyna-Gengelbach $M$, Deutschmann N, et al. Gene expression profiles in human non-small and small-cell lung cancers. Eur J Cancer 2003;39:1936-47.

17. Abba MC, Hu Y, Sun H, Drake JA, Gaddis S, Baggerly K, et al. Gene expression signature of estrogen receptor alpha status in breast cancer. BMC Genomics 2005;6:37.

18. Turtoi A, Musmeci D, Wang Y, Dumont B, Somja J, Bevilacqua G, et al. Identification of novel accessible proteins bearing diagnostic and therapeutic potential in human pancreatic ductal adenocarcinoma. J Proteome Res 2011;10:4302-13.

19. Dumont B, Castronovo V, Peulen O, Blétard N, Clézardin P, Delvenne $P$, et al. Differential proteomic analysis of a human breast tumor and its matched bone metastasis identifies cell membrane and extracellular for Scientific Research (FNRS, Belgium) and TELEVIE as well as from the Centre Anti-Cancéreux of the ULG.

The costs of publication of this article were defrayed in part by the payment of page charges. This article must therefore be hereby marked advertisement in accordance with 18 U.S.C. Section 1734 solely to indicate this fact.

Received April 22, 2013; revised June 7, 2013; accepted June 22, 2013; published OnlineFirst July 17, 2013.

proteins associated with bone metastasis. J Proteome Res 2012;11: 2247-60.

20. Bonnomet A, Syne L, Brysse A, Feyereisen E, Thompson EW, Noël A, et al. A dynamic in vivo model of epithelial-to-mesenchymal transitions in circulating tumor cells and metastases of breast cancer. Oncogene 2012;31:3741-53.

21. Turtoi A, Dumont B, Greffe $Y$, Blomme A, Mazzucchelli G, Delvenne P, et al. Novel comprehensive approach for accessible biomarker identification and absolute quantification from precious human tissues. J Proteome Res 2011;10:3160-82.

22. Turtoi A, De Pauw E, Castronovo V. Innovative proteomics for the discovery of systemically accessible cancer biomarkers suitable for imaging and targeted therapies. Am J Pathol 2011;178: 12-8.

23. Grände M, Franzen A, Karlsson JO, Ericson LE, Heldin NE, Nilsson M. Transforming growth factor-beta and epidermal growth factor synergistically stimulate epithelial to mesenchymal transition (EMT) through a MEK-dependent mechanism in primary cultured pig thyrocytes. J Cell Sci 2002;115 (Pt 22):4227-36.

24. Lee MY, Chou CY, Tang MJ, Shen MR. Epithelial-mesenchyma transition in cervical cancer: correlation with tumor progression, epidermal growth factor receptor overexpression, and snail up-regulation. Clin Cancer Res 2008;14:4743-50.

25. Price JT, Tiganis T, Agarwal A, Djakiew D, Thompson EW. Epiderma growth factor promotes MDA-MB-231 breast cancer cell migration through a phosphatidylinositol $3^{\prime}$-kinase and phospholipase C-dependent mechanism. Cancer Res 1999;59:5475-8.

26. Parton RG, Simons K. The multiple faces of caveolae. Nat Rev Mol Cell Biol 2007;8:185-94.

27. Williams TM, Lisanti MP. Caveolin-1 in oncogenic transformation, cancer, and metastasis. Am J Physiol Cell Physiol 2005;288: C494-506.

28. Lajoie P, Partridge EA, Guay G, Goetz JG, Pawling J, Lagana A, et al. Plasma membrane domain organization regulates EGFR signaling in tumor cells. J Cell Biol 2007;179:341-56.

29. Li L, Yang G, Ebara S, Satoh T, Nasu Y, Timme TL, et al. Caveolin-1 mediates testosterone-stimulated survival/clonal growth and promotes metastatic activities in prostate cancer cells. Cancer Res 2001;61:4386-92.

30. Tahir SA, Yang G, Ebara S, Timme TL, Satoh T, Li L, et al. Secreted caveolin-1 stimulates cell survival/clonal growth and contributes to metastasis in androgen-insensitive prostate cancer. Cancer Res 2001 61:3882-5.

31. Ho CC, Huang PH, Huang HY, Chen YH, Yang PC, Hsu SM. Upregulated caveolin-1 accentuates the metastasis capability of lung adenocarcinoma by inducing filopodia formation. Am J Pathol 2002; 161:1647-56.

32. Alwan HA, van Zoelen EJ, van Leeuwen JE. Ligand-induced lysosoma epidermal growth factor receptor (EGFR) degradation is preceded by proteasome-dependent EGFR de-ubiquitination. J Biol Chem 2003 278:35781-90.

33. Longva KE, Blystad FD, Stang E, Larsen AM, Johannessen LE, Madshus $\mathrm{IH}$. Ubiquitination and proteasomal activity is required for transport of the EGF receptor to inner membranes of multivesicular bodies. J Cell Biol 2002;156:843-54.

34. Roepstorff K, Grandal MV, Henriksen L, Knudsen SL, Lerdrup M, Grøvdal L, et al. Differential effects of EGFR ligands on endocytic sorting of the receptor. Traffic 2009;10:1115-27. 
35. Schmidt-Glenewinkel H, Reinz E, Bulashevska S, Beaudouin J, Legewie S, Alonso A, et al. Multiparametric image analysis reveals role of Caveolin1 in endosomal progression rather than internalization of EGFR. FEBS Lett 2012;586:1179-89.

36. Sewing A, Wiseman B, Lloyd AC, Land H. High-intensity Raf signal causes cell cycle arrest mediated by p21Cip1. Mol Cell Biol 1997; 17:5588-97.

37. Rush JS, Quinalty LM, Engelman L, Sherry DM, Ceresa BP. Endosomal accumulation of the activated epidermal growth factor receptor (EGFR) induces apoptosis. J Biol Chem 2012;287:712-22.
38. Ruth Li. The expression and effect of Myoferlin depletion in breast cancer cells [dissertation]. Columbus (OH): Ohio State University;2012.

39. Chakraborty J, Banerjee S, Ray P, Hossain DM, Bhattacharyya S Adhikary A, et al. Gain of cellular adaptation due to prolonged p53 impairment leads to functional switchover from p53 to p73 during DNA damage in acute myeloid leukemia cells. J Biol Chem 2010;285: 33104-12.

40. Kok KH, Lei T, Jin DY. siRNA and shRNA screens advance key understanding of host factors required for HIV-1 replication. Retrovirology 2009;27:6-78. 


\section{Cancer Research}

The Journal of Cancer Research (1916-1930) | The American Journal of Cancer (1931-1940)

\section{Myoferlin Is a Key Regulator of EGFR Activity in Breast Cancer}

Andrei Turtoi, Arnaud Blomme, Akeila Bellahcène, et al.

Cancer Res 2013;73:5438-5448. Published OnlineFirst July 17, 2013.

Updated version Access the most recent version of this article at: doi:10.1158/0008-5472.CAN-13-1142

Supplementary Access the most recent supplemental material at:

Material http://cancerres.aacrjournals.org/content/suppl/2013/07/16/0008-5472.CAN-13-1142.DC1.html

Cited Articles This article cites by 39 articles, 19 of which you can access for free at:

http://cancerres.aacrjournals.org/content/73/17/5438.full.html\#ref-list-1

Citing articles This article has been cited by 1 HighWire-hosted articles. Access the articles at: http://cancerres.aacrjournals.org/content/73/17/5438.full.html\#related-urls

E-mail alerts Sign up to receive free email-alerts related to this article or journal.

Reprints and To order reprints of this article or to subscribe to the journal, contact the AACR Publications Department at Subscriptions pubs@aacr.org.

Permissions To request permission to re-use all or part of this article, contact the AACR Publications Department at permissions@aacr.org. 